\title{
Patterns of schematic structure and strategic features in newspaper editorials: a comparative study of American and Malaysian editorials
}

\begin{abstract}
To carry a message through effectively to the public, newspaper editors need to employ the generic pattern of editorials as a rule of thumb. Yet few studies have investigated the schematic structure and persuasive style of editorials. Hence, this study aims to compare the generic characteristics in 240 editorials of The New York Times $(N Y T, n=120)$ and New Straits Times (NST, $n=120$ ). To realize the objectives, the corpus was subjected to a content analysis based on a composite framework drawn from the data and previous models. The findings revealed that American and Malaysian editorials share a similar schematic structure at the move level including four obligatory moves. However, at the step level, evidence of disparity of the style of writing was apparent. The data obtained could be used as informed input in the English for Specific Purposes (ESP) classroom, so that English as a Secondary Language (ESL)/English as a Foreign Language (EFL) students' awareness on the conventional structures in editorial writing could be heightened.
\end{abstract}

Keyword: Schematic structure; Persuasive style; Newspaper editorials; Comparative study; American editorials; Malaysian editorials 